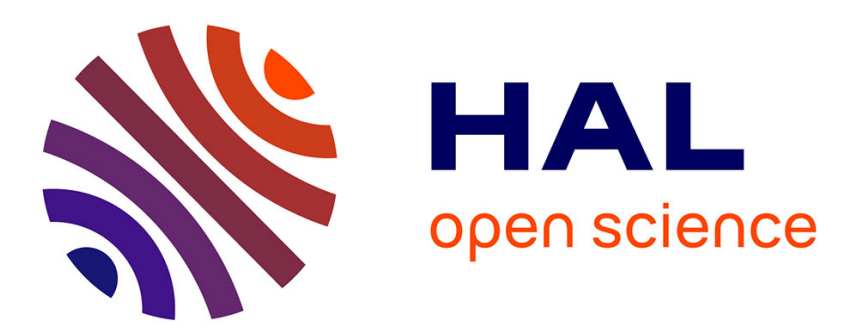

\title{
In-Situ SEM Study of Fatigue Crack Growth Mechanism in Carbon Black filled Natural Rubber
}

\author{
Stéphanie Beurrot, Bertrand Huneau, Erwan Verron
}

\section{To cite this version:}

Stéphanie Beurrot, Bertrand Huneau, Erwan Verron. In-Situ SEM Study of Fatigue Crack Growth Mechanism in Carbon Black filled Natural Rubber. Journal of Applied Polymer Science, 2010, 117 (3), pp.1260-1269. 10.1002/app.31707 . hal-01006960

\section{HAL Id: hal-01006960 https://hal.science/hal-01006960}

Submitted on 29 Sep 2017

HAL is a multi-disciplinary open access archive for the deposit and dissemination of scientific research documents, whether they are published or not. The documents may come from teaching and research institutions in France or abroad, or from public or private research centers.
L'archive ouverte pluridisciplinaire HAL, est destinée au dépôt et à la diffusion de documents scientifiques de niveau recherche, publiés ou non, émanant des établissements d'enseignement et de recherche français ou étrangers, des laboratoires publics ou privés. 


\title{
In Situ SEM Study of Fatigue Crack Growth Mechanism in Carbon Black-Filled Natural Rubber
}

\author{
Stéphanie Beurrot, Bertrand Huneau, Erwan Verron \\ Institut de Recherche en Génie Civil et Mécanique (GeM), UMR CNRS 6183, Ecole Centrale de Nantes, \\ BP 92101, 44321 Nantes cedex 3, France
}

\begin{abstract}
A micro-tensile testing machine placed in the chamber of a scanning electron microscope is used to perform in situ fatigue tests on a 43 phr carbon black-filled cis-1,4-polyisoprene rubber; the crack tip is observed in real-time during crack propagation. These observations lead to a detailed description of the crack tip morphology; the crack front is a regular pattern of diamond-shaped zones delimited by extended straight ligaments. Fatigue crack growth is driven by the ligaments breakage, which occurs in all the zones of the crack front surface continuously in time but at different velocities. This phenomenon of nonlocalized damage explains initia-
\end{abstract}

tion and limited propagation of branches which deviate from the main crack. All those mechanisms are sources of energy dissipation which explains the great fatigue properties of NR. Finally, from similar experiments conducted on styrene butadiene rubber, it is established that the peculiar morphology of the crack tip and mechanism of crack propagation in NR are due to strain-induced crystallization.

Key words: elastomers; fatigue analysis; natural rubber; crack propagation; electron microscopy

\section{INTRODUCTION}

Carbon black-filled natural rubber (CB-NR) exhibits longer fatigue life than other elastomeric materials. ${ }^{1}$ Number of mechanical studies have been proposed to quantify the long-term durability of this material (see refs. ${ }^{2}$ and ${ }^{3}$ and the references herein). Nevertheless, only few recent papers investigate the microstructural aspects of fatigue crack growth in rubber ${ }^{4,5}$; in these studies, fatigue tests are first conducted until a sufficiently long fatigue crack develops in the sample, then this sample is stretched and the open crack is observed in a scanning electron microscope (SEM). Moreover, Le Cam et al. developed an original "microcutting" technique, which permits to observe fatigue damage behind the crack tip and then to propose a scenario of fatigue crack growth in NR. ${ }^{4}$

The aim of this article is to verify the previously proposed mechanism and to enrich the understanding of the phenomena involved during fatigue crack growth at the microstructural scale. For this purpose, in situ SEM fatigue experiments are conducted using a micro-tensile testing machine, and crack propagation is observed in real-time. Indeed, we believe that only such observations enable to unquestionably establish the scenario.

Correspondence to: E. Verron (erwan.verron@ec-nantes.fr).
The experimental procedure is first described in details. Then, the next section presents the thorough description of the crack tip, clarifies the scenario of crack propagation, and explains how secondary cracks appear. Finally, these results are discussed in the light of both macroscopic fatigue properties and strain-induced crystallization of natural rubber.

\section{EXPERIMENTAL}

The aim of this experiment is to observe the evolution of the crack tip during rubber fatigue crack propagation, in real-time. The experiments are conducted in three steps: (i) the specimen is precut with a scalpel; (ii) a classical fatigue experiment is then conducted in a standard machine until a fatigue crack propagates; and (iii) the experiment is continued in a micro-tensile testing machine placed in a SEM.

\section{Apparatus}

The micro-tensile testing machine is shown in Figure 1 ; it was designed by Deben UK, especially for soft materials (the distance between the clamps varies from 20 to $40 \mathrm{~mm}$ ). This machine is sufficiently small sized to be placed in the chamber of a SEM, it is monitored by a computer connected via a leakfree connector, and the double-screw driving system leads to easy observation because the center of the sample, that is, the position of the crack tip, does 


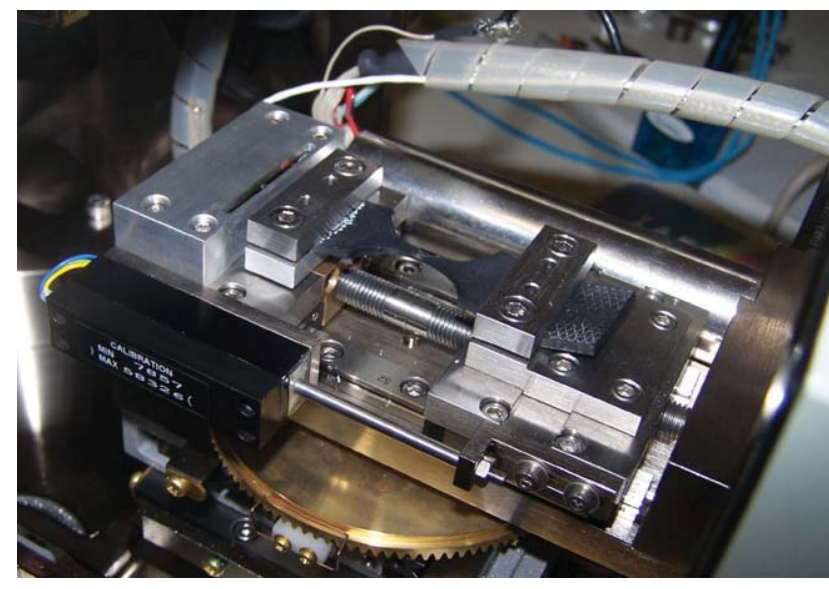

Figure 1 Micro-tensile testing machine. [Color figure can be viewed in the online issue, which is available at www.interscience.wiley.com.]

not change during loading. Nevertheless, two major difficulties are due to the characteristics of the machine. First, its size and the limited distance between the clamps constrain both size and shape of the samples. Indeed, a special design of samples is needed to induce large strain in the vicinity of the crack. Second, as the relative speed of the clamps can vary from 0.1 to $2 \mathrm{~mm} / \mathrm{min}$, the maximum frequency of a fatigue test is $0.83 \mathrm{mHz}$, such value leading to days-long experiments. This is the reason why samples are precut and the fatigue crack is first propagated in a classical tensile-testing machine.

\section{Material and design of the sample}

The material considered here is an industrial $43 \mathrm{phr}$ CB-NR, provided in 2-mm thick calendered sheets.

As mentioned earlier, a special shape of sample was chosen in order to achieve large strain in crack vicinity and to propagate it; the corresponding geometry is shown in Figure 2. To ensure that the deformation is predominantly uniaxial extension, we performed a finite element simulation of the sample (without a precut) for a 20-mm long sample stretched to $40 \mathrm{~mm}$ long. In this case, the logarithmic strain tensor in the center of the sample is as follows:

$$
\left[\begin{array}{ccc}
1.40 & 0.02 & 0 \\
0.02 & -0.71 & 0 \\
0 & 0 & -0.70
\end{array}\right]
$$

where the directions are defined in Figure 2. The first component corresponds to a stretch ratio of about $400 \%$ and the shear components are negligible, so the deformation at crack tip is nearly uniaxial.

\section{Procedure}

i. The first step consists in precutting the sample to localize the crack in its center. This cut is made with a scalpel and the resulting crack is $<1 \mathrm{~mm}$ deep and between $2 \mathrm{~mm}$ and $3 \mathrm{~mm}$ long.

ii. To reduce the duration of the fatigue experiment, a fatigue crack is initiated from the precut in a standard fatigue machine. Fully relaxing cycles of stretch amplitude 200\% are considered and the strain rate is chosen to limit self-heating, in order to not superimpose thermal damage to mechanical damage. Practically, it corresponds to frequencies $<1 \mathrm{~Hz}$. Finally, this first part of the fatigue test performed in a standard machine is stopped after the crack has propagated of about $50 \%$ of precut deepness.

iii. Finally, the experiment is continued in the small-scale tensile machine. Loading conditions are identical to those of (ii), except the frequency which is $0.83 \mathrm{mHz}$. Observation is made in a JEOL 6060LA SEM using secondary electrons imaging. Note that if a too high power electron beam is used, crack tip is damaged: microscopic cracks develop perpendicular to the loading direction. To overcome this difficulty, four parameters need to be lowered to reduce the energy of the electron beam per unit area: the probe current, the accelerating voltage, the magnification, and the exposure time. Once these parameters set, the fatigue experiment is stopped once per cycle at maximum stretch to photograph the crack tip.

\section{Remark on the validity of the experimental method}

We recall that the objective of the experiments is to establish the fatigue crack growth mechanism in ambient air at conventional frequencies (about $1 \mathrm{~Hz}$ ) and that in situ observations cannot be conducted with those experimental conditions. So, an essential prerequisite is to check that the mechanism is not affected by this change of experimental conditions. First, assuming that a given mechanism always leads to similar crack tip morphologies, we verified that crack tips before and after in situ fatigue tests have the same characteristics: similar microstructural features with the same sizes and proportions. Second, we verified a posteriori that the change in experimental conditions does not influence the mechanism by examining the fracture surface of the samples: the transition between the precut step (i) 


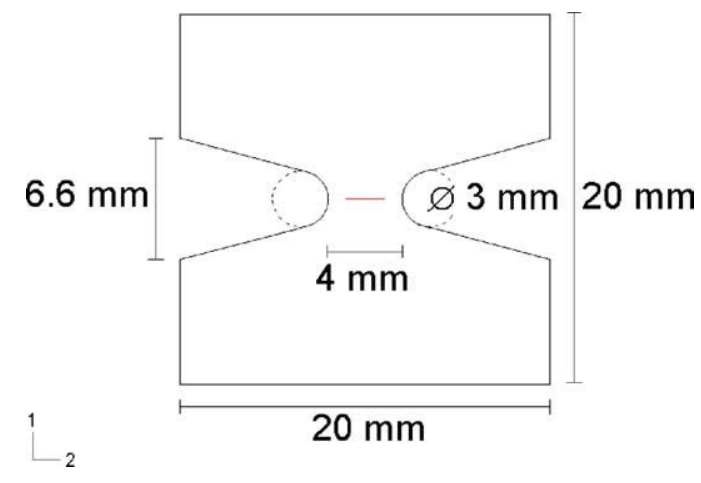

Figure 2 Geometry of the samples. [Color figure can be viewed in the online issue, which is available at www. interscience.wiley.com.]

and the conventional fatigue step (ii) is visible, but the one between conventional (ii) and in situ (iii) fatigue tests is not.

\section{MECHANISM OF FATIGUE CRACK PROPAGATION}

\section{Description of the crack tip}

Figures 3 and 4 present the front view and the side view, respectively, of the fatigue crack tip. In the former figure, tensile direction is indicated by white arrows and the propagation direction is normal to the photomicrograph. As observed previously, ${ }^{4,5}$ the crack tip is composed of number of diamond-shaped zones separated by ligaments. The pattern of liga-

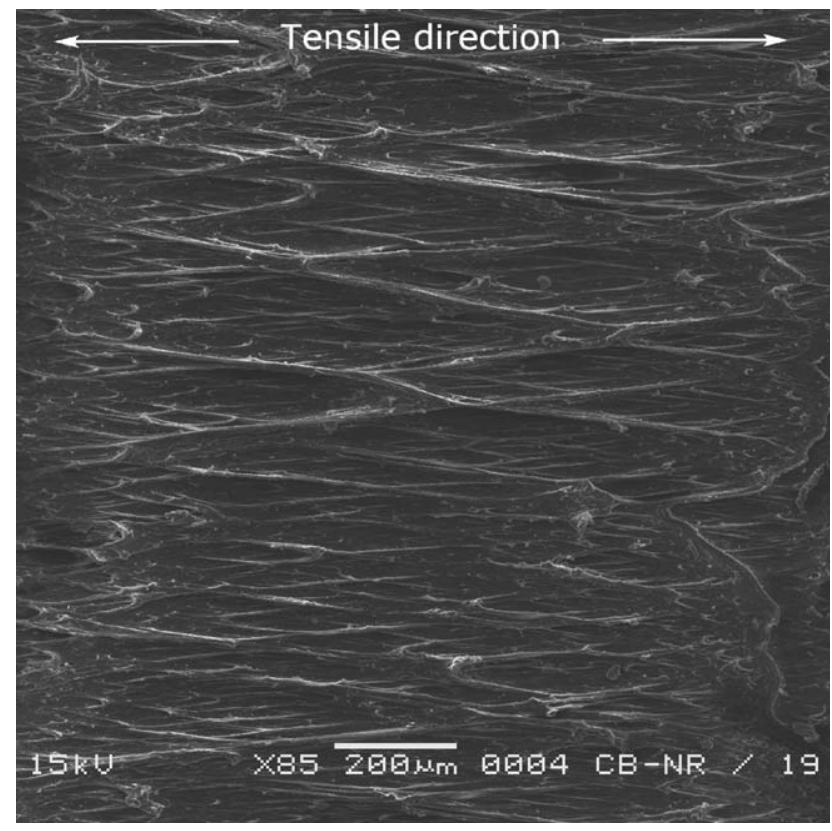

Figure 3 Top view of a fatigue crack tip.

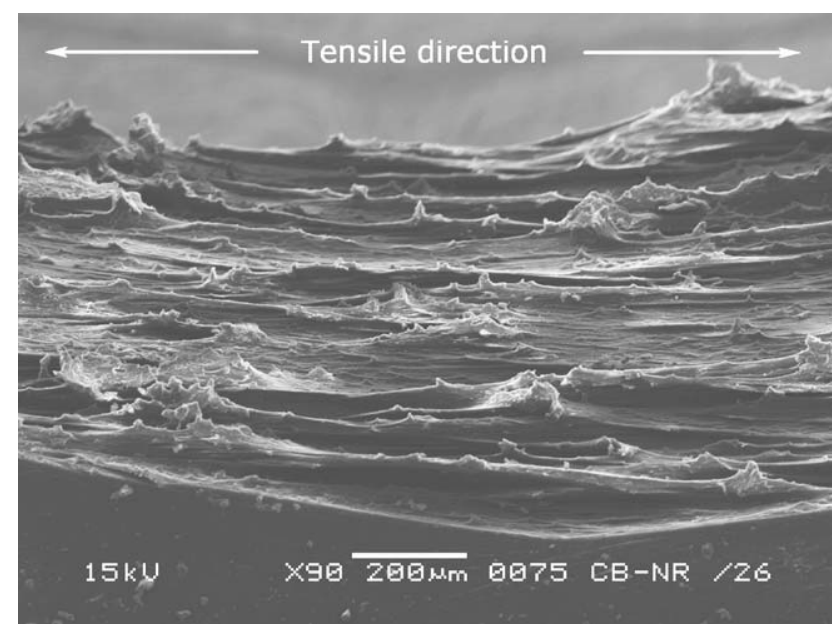

Figure 4 Side view of a fatigue crack tip.

ments and diamond-shaped zones can be described as multiscaled because large diamond-shaped zones delimited by large ligaments are themselves made up of smaller zones delimited by smaller ligaments. Figure 4 shows that the crack tip is a "hilly landscape": the diamond-shaped zones are flat and smooth compared with the ligaments, and those ligaments emerge from the smooth surfaces. The most noticeable characteristic of the crack tip is the pattern regularity. Surprisingly, the ligaments are not parallel to the tensile direction. In fact, they are parallel to two directions which are symmetric with respect to the tensile direction. The angle between the direction of ligaments and the loading direction decreases with the extension of the sample. Consequently, at a given deformation even if the size of diamond-shaped zones varies, all of them have the same length-width ratio. For instance, in Figure 3, this ratio is 4 to 1 . Nevertheless, the size of the diamond-shaped zones is not uniform; indeed the knots of the pattern are not regularly located.

Then, we examine more precisely the ligaments. They vary in shape and size. First, they can be compared in regard to their size: (1) large ligaments delimit large diamond-shaped zones, (2) intermediate-sized ligaments delimit small diamond-shaped zones, and finally, (3) the smallest ligaments are those contained in the small diamond-shaped zones. Second, ligaments can also be compared in regard to their shape. It leads to four types of ligaments defined by the form of their section as shown in Figure 5: those with uniform width along their height (mostly large ligaments) [Fig. 5(a)], those with a large base (mostly intermediate-sized and small ligaments) [Fig. 5(b)], those with a narrow base (large and intermediate-sized ligaments) [Fig. 5(c)], and finally, those which form a bridge (very rare, small ligaments) [Fig. 5(d)]. Finally, the crack tip also 

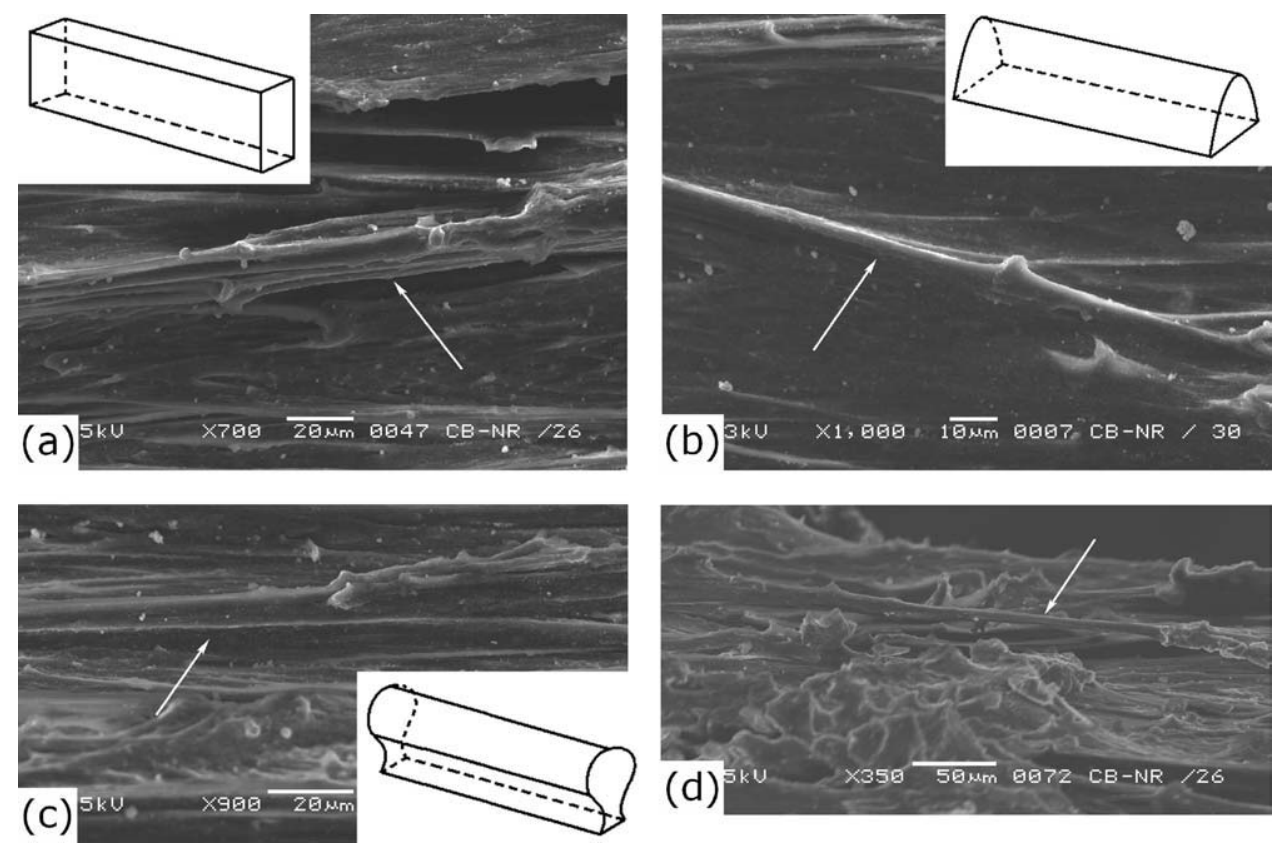

Figure 5 SEM pictures and schemes of the four main ligament shapes: (a) ligament with uniform width, (b) ligament larger at the base, (c) ligament larger at the top, and (d) ligament shaped as a bridge.

contains another relief feature shown in Figure 6. As it will be established in the following, it corresponds to previously broken and then shrunk ligaments. They are located at the knots of the pattern, that is, at the intersection of the ligaments.

The previous description was devoted to rubber matrix, but as the material considered in this study is an industrial CB-NR, it contains a lot of different inclusions. Most of them are zinc oxides, in both diamond-shaped zones and ligaments, or carbon black agglomerates in diamond-shaped zones. In most of the cases, they are contained in elliptical cavities.

Finally, Figure 7 summarizes the description of the crack tip microstructure: the left-hand drawing shows the front view and presents the different elements described above and the right-hand drawing is a side view which highlights the relief of the crack front. Table I gives the mean sizes and quantities of those features measured on a $500 \mu \mathrm{m} \times 800 \mu \mathrm{m}$ area of a given crack tip. The sample and the area were chosen for their representativeness.

\section{How does the crack propagate?}

Figure 8 shows six SEM images of a $0.5 \mathrm{~mm}^{2}$ area of the crack tip taken for the maximum stretch of in situ fatigue cycles 1, 10, 21, 31, 41, and 51, respectively. The micromechanism of fatigue crack growth in rubber can be established using Figure 8. From one SEM image to another, it clearly appears that all the crack tip zones are affected by crack growth; positions of ligaments change with loading as shown by the white lines drawn on images. It means that the crack front is a surface rather than a line. Moreover, the evolution of the diamond-shaped zones suggests a three-step mechanism for crack propagation as emphasized in Figure 9. To describe this evolution, we choose Figure 9(a) as the reference state of the zones. Under loading, zones become larger [see changes between Fig. 9(a-c)], and cavities and inclusions appear on the surface [see Fig. 9(c)]. It means that the matter tears and the zone grows deeper in the direction normal to the crack surface, that is, the direction of crack growth (it cannot be seen in the figure). The more the zone goes deeper,

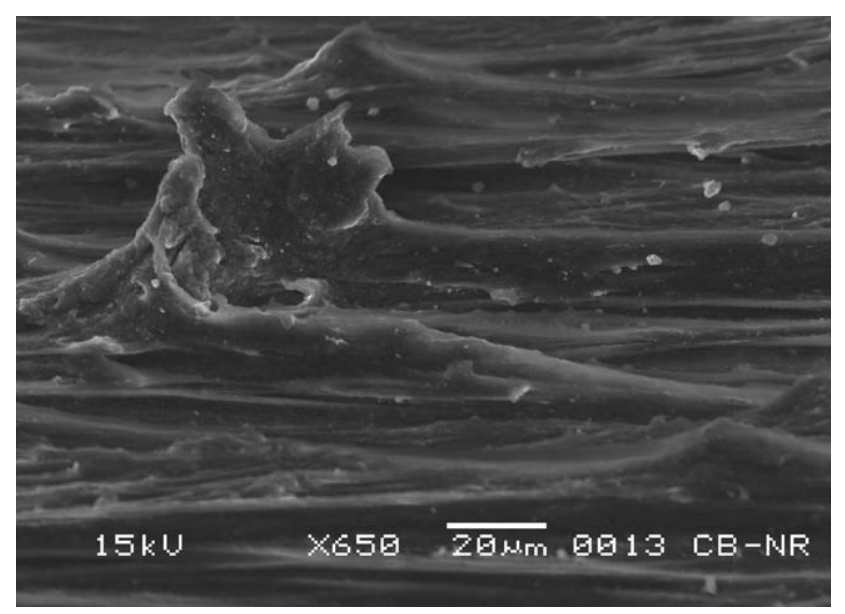

Figure 6 Example of a shrunk ligament. 


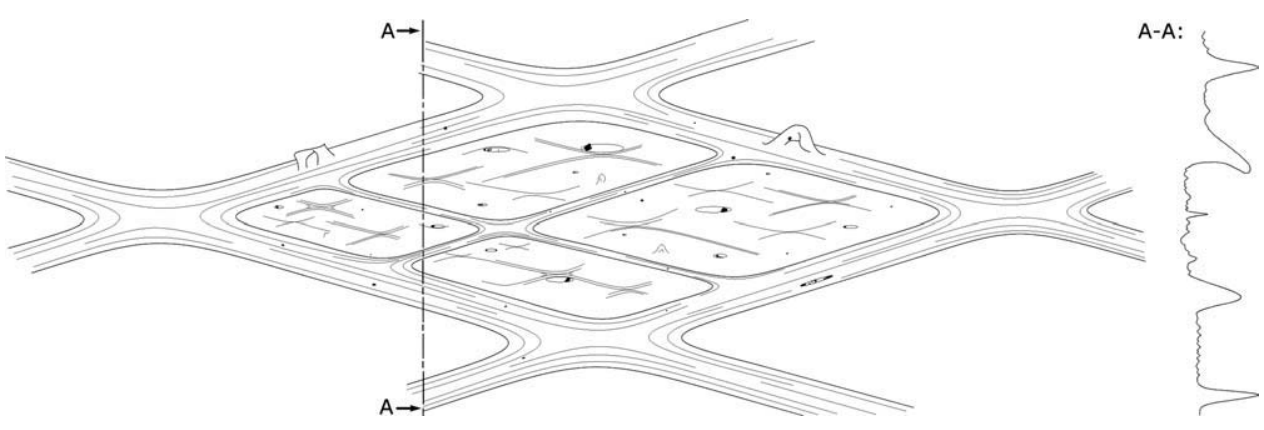

Figure 7 Open crack tip (top view and section).

the more the ligaments which delimit it are stretched. Then, after a few number of cycles, one of these ligaments breaks [see Fig. 9(d)] and shrinks [see Fig. 9(e)]. This breakage is not instantaneous: it lasts from 1 to 10 cycles depending on the size and shape of the ligament and of the local microstructure. Two different evolutions of the microstructure are observed: a new ligament may emerge or the two diamond-shaped zones separated by the broken ligament may coalesce as shown in Figure 9(f). In both cases, the matter reorganizes itself in a large vicinity of the broken ligament through the displacement of ligaments and diamond-shaped zones. In fact, this mechanism repeats itself in every diamondshaped zone of the crack front. From a temporal point of view, this phenomenon occurs in a continuous manner with different velocities in each point. From a spatial point of view, it can happen simultaneously in different locations of the crack front.
To close this section, we summarize the main elements of the previous mechanism with the help of a five-step scenario as depicted in Figure 10.

a. Figure 10(a) presents the initial state of a diamond-shaped zone and its delimiting ligaments; it is a detail of the drawing of the crack tip in Figure 7,

b. The diamond-shaped zone grows larger and deeper, showing new inclusions and ligaments [see Fig. 10(b)],

c. As a consequence, ligaments which delimit the zone are stretched and one of them gets thinner than in the previous cycle [see Fig. 10(c)],

d. Eventually, this ligament breaks [see Fig. 10(d)], and

e. Finally, the matter reorganizes itself through coalescence of the diamond-shaped zone with one of its neighbors [see Fig. 10(e)].

TABLE I

Sizes and Quantities of the Elements Measured on a $500 \mu \mathrm{m} \times 800 \mu \mathrm{m}$ Area of a Given Crack Tip

\begin{tabular}{lll}
\hline \multicolumn{1}{c}{ Element type } & \multicolumn{1}{c}{ Mean size $\left(\mu \mathrm{m}\right.$ or $\mu \mathrm{m}^{2}$ or $\left.\mu \mathrm{m}^{3}\right)$} & Quantity observed on the chosen sample \\
\hline Large diamond-shaped zones & $200 \times 800$ & 4 \\
Small diamond-shaped zones & $100 \times 400$ & 20 (from 3 to 8 per large zone) \\
Large ligaments & (Width $\times$ height $\times$ length) $20 \times 50 \times 600$ & 4 \\
Intermediate-sized ligaments & (Width $\times$ height $\times$ length) $10 \times 25 \times 300$ & Around 100 \\
Small ligaments & (Width $\times$ height $\times$ length) $1 \times 5 \times 50$ & Several hundreds \\
Zinc oxides & Diameter: $<10$ (exceptionally 20) & Several hundreds \\
Cavities around zinc oxides & Elliptical: $7 \times 20$ & Several tens \\
Carbon black agglomerates & Diameter: 50 & 1 \\
Cavities around agglomerates & Elliptical: $50 \times 200$ & 1 \\
Shrunk ligaments & Diameter: from 1 to 100 & Around 100 \\
Ligaments with uniform width & - & $2 / 3$ of all large ligaments, $1 / 4$ of \\
& & all intermediate-sized ligaments, \\
& - & $1 / 4$ of all small ligaments \\
Ligaments with a large base & - & $1 / 2$ of all intermediate-sized ligaments, \\
& $3 / 4$ of all small ligaments \\
Ligaments with a narrow base & - & $1 / 3$ of all large ligaments, $1 / 4$ of all in \\
"Bridge" ligaments & termediate-sized ligaments \\
& & $1 \%$ of all small and intermediate-sized \\
& ligaments
\end{tabular}



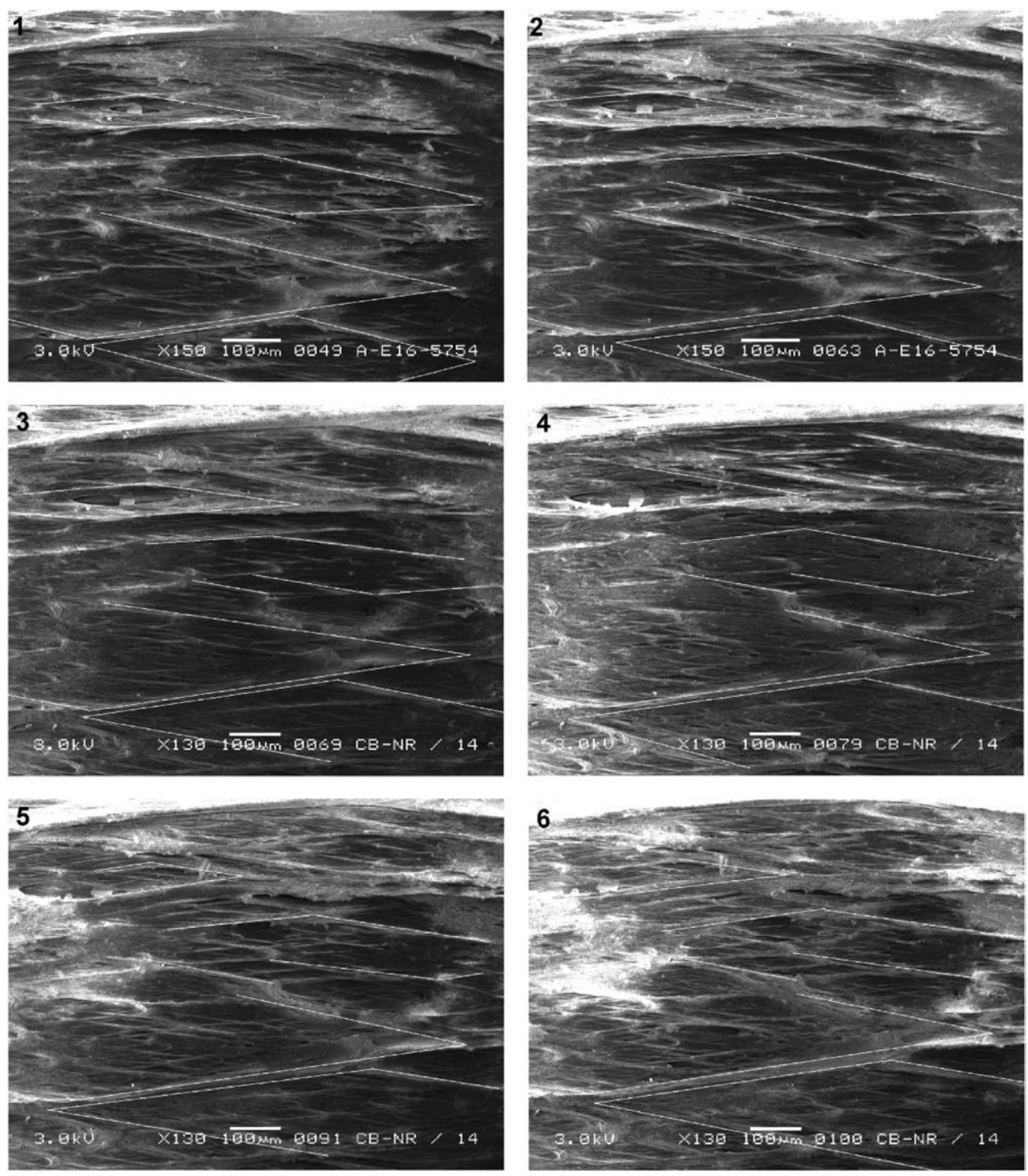

Figure 8 Evolution of a crack tip during cyclic loading after 1, 10, 21, 31, 41, and 51 in situ cycles, respectively.

\section{How do secondary cracks appear?}

In the previous sections, the crack was observed only when opened. However, the study of the closed crack exhibits the path of a fatigue crack in natural rubber. To perform these observations, samples are cut in two with a scalpel along the plane defined by the tensile and propagation directions in the middle of the crack as shown in Figure 11. Figure 12 presents the side view of the crack, which is slightly open to observe more easily the crack path. It highlights the main crack path which is normal to the tensile direction and several secondary cracks. As in Hamed, ${ }^{6}$ the term "secondary crack" refers to short deviated cracks developed from the main one. Their length varies from 10 to $100 \mu \mathrm{m}$. To establish the scenario of secondary cracks formation also called "crack branching phenomenon," it is necessary to determine how diamond-shaped zones evolve rela- tively to the others. In this purpose, the crack tip is observed with a different orientation from the previous images (Figs. 8 and 9); samples are rotated $90^{\circ}$ in the SEM chamber to change the orientation of the ligaments with respect to the secondary electron detector. The obtained image is presented in Figure 13; it emphasizes the relief of the crack tip due to the shadow contrast. It shows that all diamond-shaped zones are not in the same plane, some of them are deeper than others (see those indicated by white arrows in Fig. 13).

The crack propagation mechanism proposed in Section "How does the crack propagate?" does not take into account kinetics. The presence of diamond-shaped zones of different deepness in Figure 13 suggests that even though the diamond-shaped zones evolve in a continuous manner, they do not grow deeper at the same speed. Moreover, recalling 

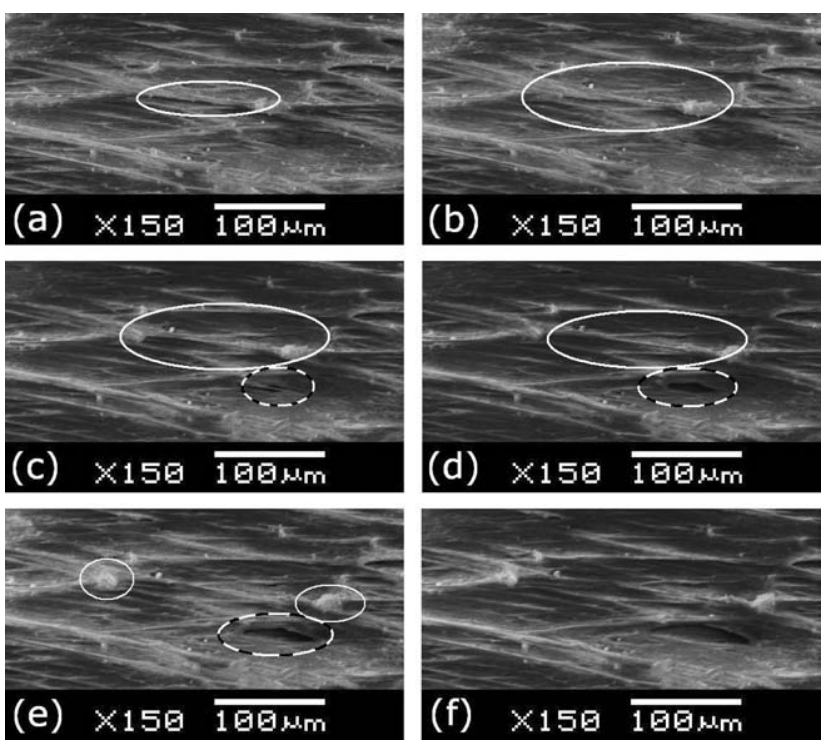

Figure 9 Successive photographs of the same detail of a crack tip during the rupture of a ligament after $1,3,5,6$, and 8 in situ cycles, respectively.

that the crack front is a surface rather than a line, the crack branching scenario will be established with the help of Figure 14. It shows the evolution under fatigue loading of three contiguous diamondshaped zones ((a), (b), and (c)) located at the crack front surface. It is a simplified representation of the crack tip cross-section: the right-hand drawings describe the close crack with the same view as in Figure 12 and the left-hand drawings show the same crack but open. To simplify the discussion, the scenario is established by considering three successive steps.

Initially, the three diamond-shaped zones (a), (b), and (c) are separated by ligaments (grey in the figure) and have the same depth. When the crack is closed, there is only one branch.

Later on, when the crack has propagated, zones (a) and (c) have grown deeper than zone (b). So, the close crack presents two similar branches. The main crack will develop from one or the other ((a) or (c)).

As the crack continues to propagate, one of the two previous branches will become the main crack due to both local mechanical conditions and microstructure. In this figure, zone (a) has become the main crack, and turned into two new diamondshaped zones (e) and (f) because the former ligaments which delimited zone (a) broke and new ones appeared; zone (b) does not evolve anymore because it is partially relaxed; and zone (c) forms a secondary crack.

During crack propagation, the elementary scenario described above repeats: zones (e) and (f) will evolve in the same manner as zones (a), (b), and (c) in step
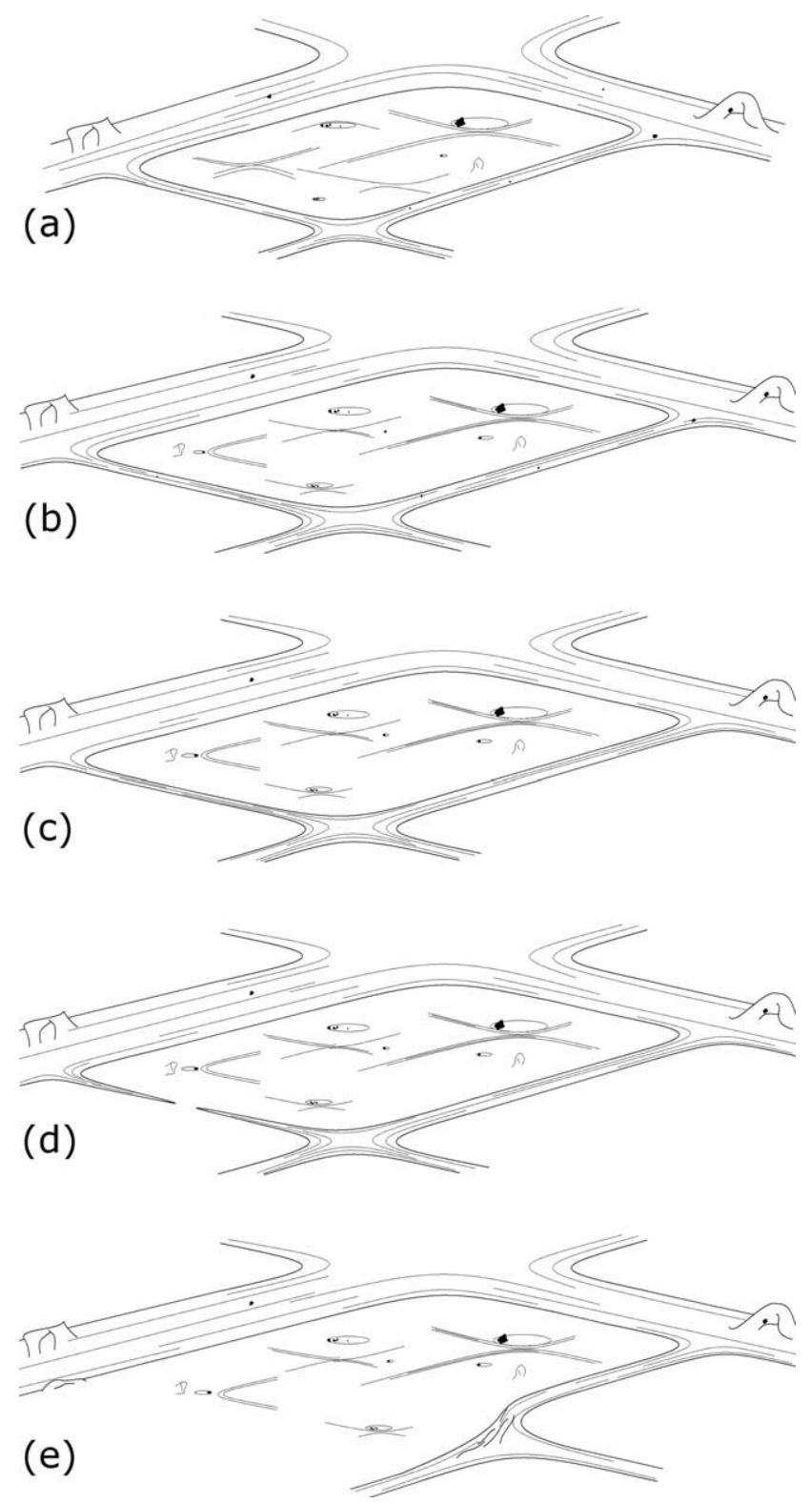

Figure 10 Fatigue crack propagation mechanism.

(1). It is to note that zones (a), (b), and (c) can be three single diamond-shaped zones or three groups of several zones.

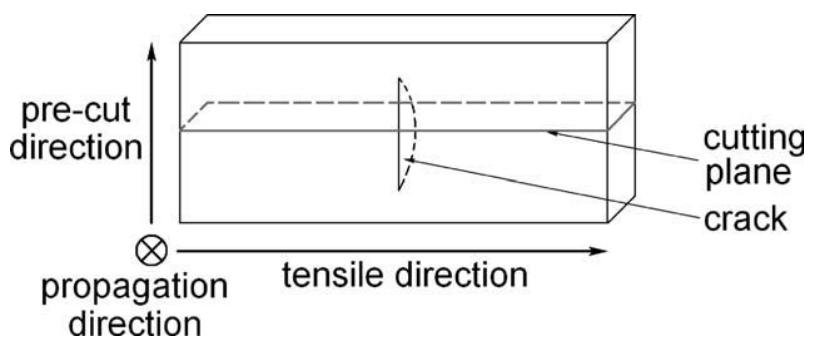

Figure 11 Cutting plane of the specimens after fatigue tests. 


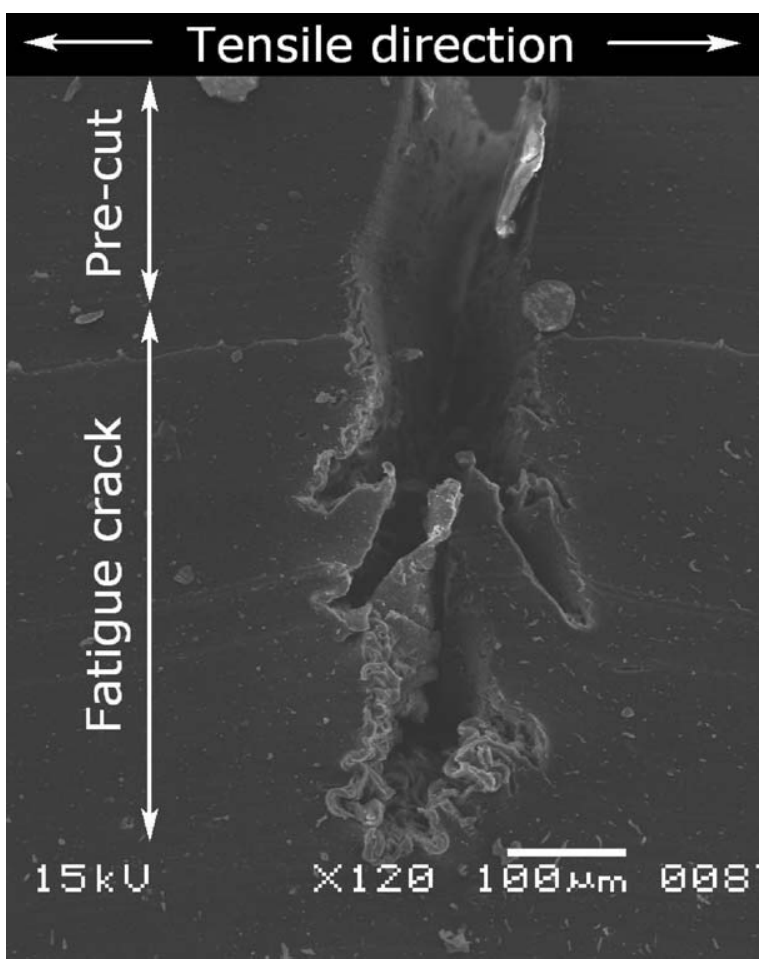

Figure 12 Crack path observed in a cut sample.

Finally, the crack branching scenario enables us to explain the roughness of the fracture surface morphology shown in Figure 15. More precisely, the fracture surface contains numerous "leaves," that is, thin pieces of matter orientated toward the initiation zone of the crack; their size varies between $10 \mu \mathrm{m}$ and $100 \mu \mathrm{m}$ similarly to the length of secondary cracks. In fact, the matter which separates secondary cracks from the main crack when we observe the close crack corresponds to the leaves on the fracture

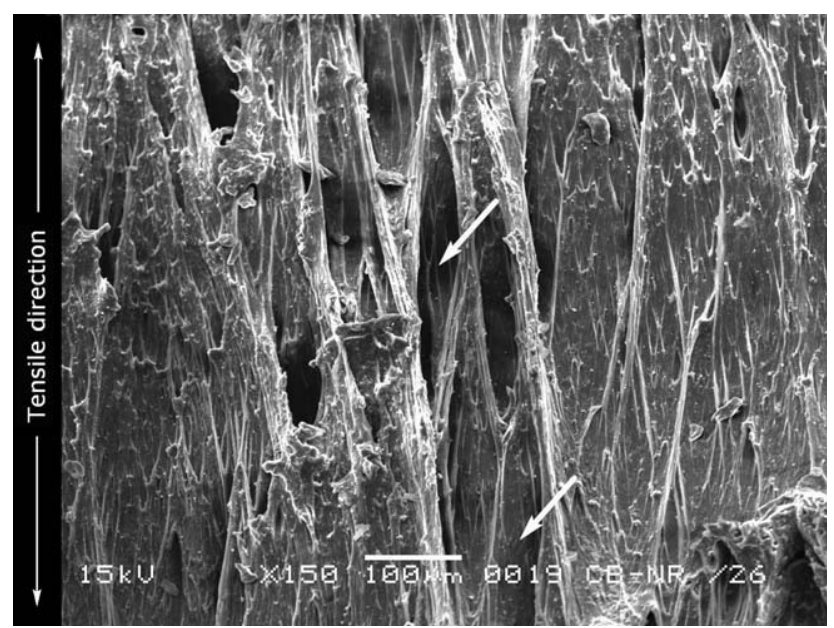

Figure 13 Top view of a crack tip in a $90^{\circ}$ rotated specimen in the chamber of the specimen.

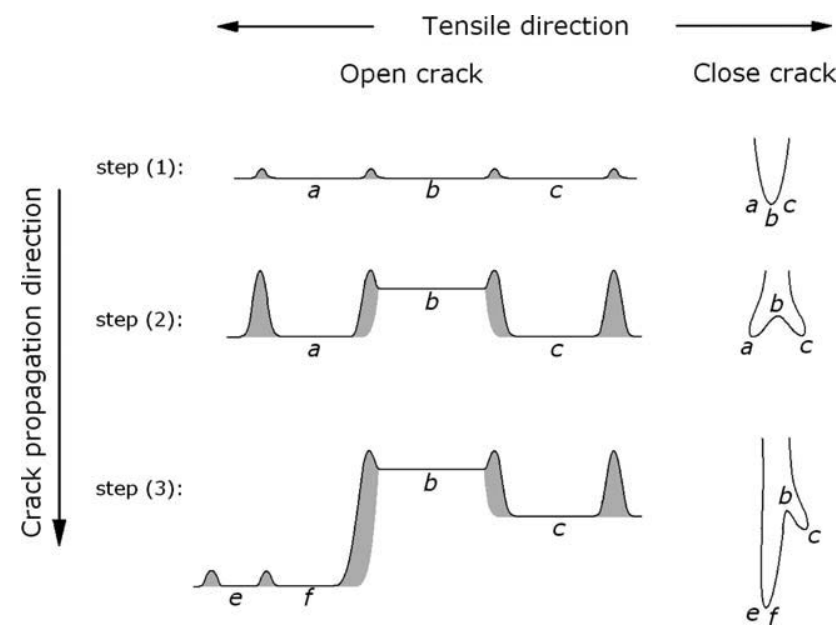

Figure 14 Branching phenomenon mechanism.

surface; this is shown in Figure 16, Figure 16(a) is the sketch of the close crack shown in Figure 12 and Figure 16(b) is a sketch of the side view of the fracture surface in Figure 15.

\section{DISCUSSION}

Recently, some authors performed interrupted fatigue tests and observed stretched samples in SEM. ${ }^{4,5}$ Le Cam et al. observed the damage induced by fatigue and proposed the mechanism of crack propagation in natural rubber. Nevertheless, only observations performed during crack propagation enable to unquestionably establish this mechanism. Here, in situ SEM experiments have been conducted to observe fatigue crack propagation in rubber in realtime. If such experiments have been already conducted for metallic materials, ${ }^{7}$ to our knowledge, this work is the first attempt to apply this technique to

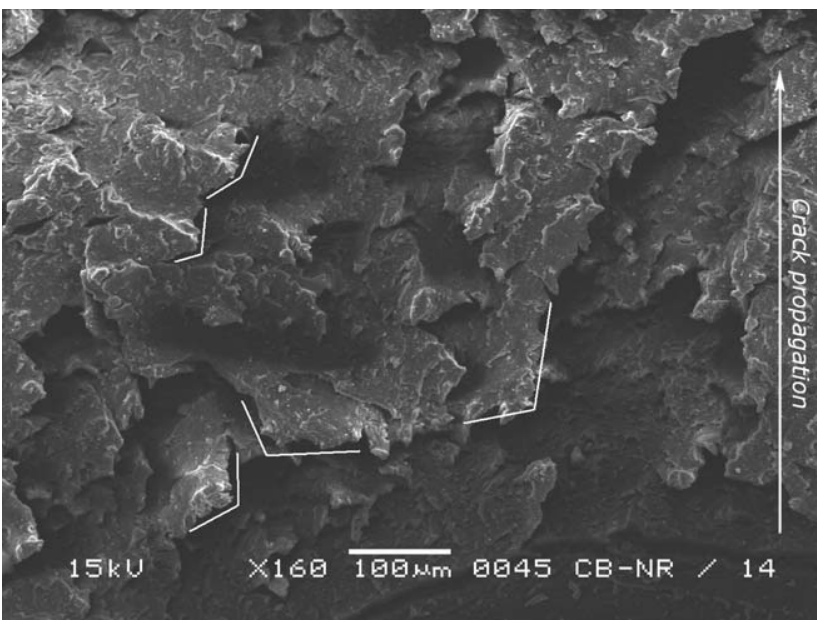

Figure 15 Detail of the fracture surface (top view). 


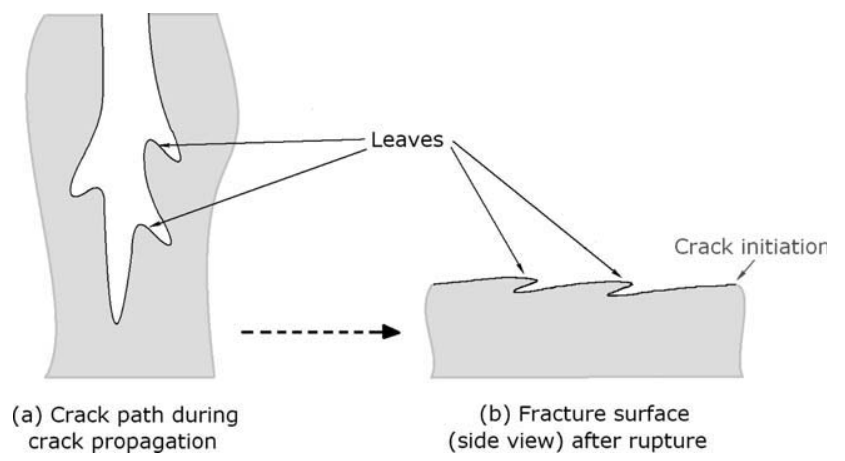

Figure 16 Origin of the fracture surface leaves.

rubber materials. Some experimental difficulties have been overcome. These difficulties are mainly due to the nonconductive nature of polymers: first, the build-up charge leads to low-quality images, and second, the electron beam causes radiation damage which alters the material. It can lead to false interpretation as underlined by White and Thomas. ${ }^{8}$ The classical way to counter these difficulties consists in applying a conductive coating to the sample; in our case, this solution could not be applied because the coating layer would have been destroyed by cyclic loading. As detailed in the Procedure section, we reduce the energy of the electron beam per unit area.

With this careful procedure, we are able to highly improve the description of both crack tip morphology and propagation mechanism, previously published. ${ }^{4}$ First, the crack tip description is enriched; ligaments morphology and orientation were thoroughly investigated. Second, this mechanism of crack propagation is in good agreement with the previous one, except for the perpendicular microcracks and the cavities which were observed by Le Cam et al. and not here. In fact, those micro-cracks were due to the electron beam; moreover, we believe that what was called a cavity in our previous study was only a smooth hollow that is overestimated for its deepness. Third, additional experiments allow explaining both crack branching and rough surface morphology in NR under fatigue loading conditions.

The microscopic mechanism presented earlier explains the great fatigue properties of NR at the macroscopic scale: long fatigue life ${ }^{9}$ and low crack growth rate. ${ }^{2,10}$ Indeed, the ligaments of the crack tip resist to crack propagation in two ways: they induce a surface crack front rather than a tearing line as well as branched cracks which both help to dissipate energy and then hold up crack advance as previously argued by Hamed. ${ }^{6}$

The great fatigue properties of NR are usually correlated with its ability to crystallize under deformation. To relate this property to our results, we will now compare NR with styrene butadiene rubber
(SBR), an elastomer which does not exhibit straininduced crystallization. More precisely, a similar study has been conducted for a SBR material with the same amount of carbon black fillers than in the NR considered here. It highlights three main differences between NR and SBR fatigue crack growth mechanisms:

- As shown in Figure 17(a) compared with Figure 11(b), the crack tip of SBR does not present ligaments. Even though we observe filaments parallel to tensile direction, the crack tip is very smooth and experiments reveal that those filaments do not resist to crack propagation as ligaments do in NR,

- The in situ crack propagation observation also shows that in SBR the crack front is a line rather than a surface as in NR,

- The close crack in Figure 17(b) shows that crack branching does not occur in SBR contrary to what is observed in NR (see Fig. 12).

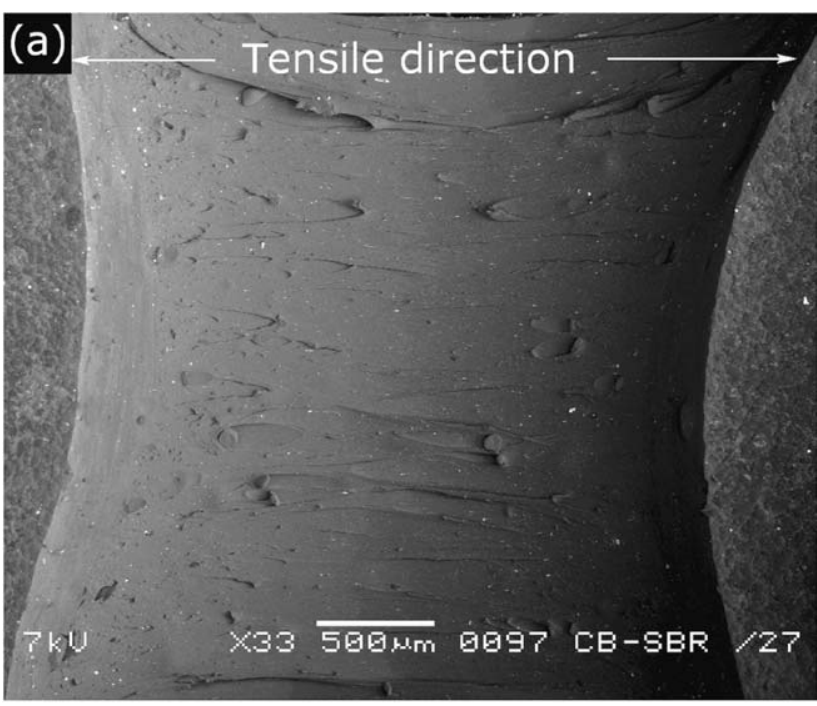

(b)

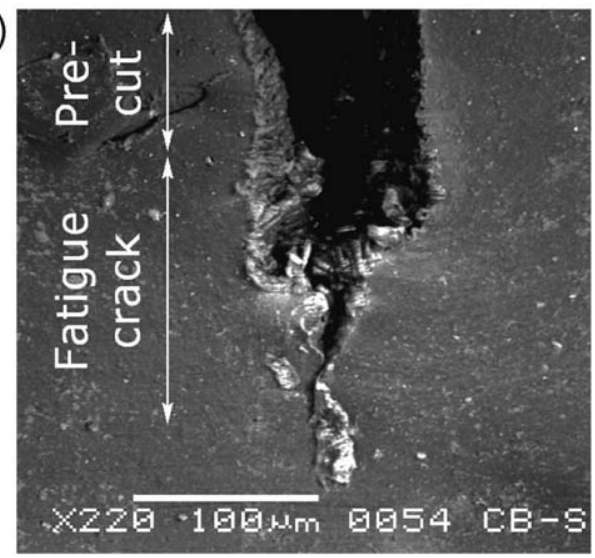

Figure 17 SBR specimen: (a) Front view of the open crack tip, (b) side view of the close crack. 
This comparison demonstrates that the heterogeneity of the microstructure at the crack tip (ligaments, diamond-shaped zones) is a consequence of strain-induced crystallization in NR.

At the close of this study, the mechanism of fatigue crack propagation in NR is qualitatively well-established; further investigations are now required to quantify the heterogeneity of some physical quantities, for example, crystallinity and strain, especially at the crack tip, which is in a quite different energy state in comparison with in bulk.

The authors thank Trelleborg Automotive for providing the material for this study.

\section{References}

1. Beatty, J. R. Rubber Chem Technol 1964, 37, 1341

2. Lake, G. J. Rubber Chem Technol 1995, 68, 435.

3. Mars, W. V.; Fatemi, A. Int J Fatigue 2002, 24, 949.

4. Le Cam, J. B.; Huneau, B.; Verron, E.; Gornet, L. Macromolecules 2004, 37, 5011.

5. Hainsworth, S. V. Polym Test 2007, 26, 60.

6. Hamed, G. R. Rubber Chem Technol 1994, 67, 529

7. Crepin, J.; Bretheau, T.; Caldemaison, D.; Ferrer, F. Acta Mater 2000, 48, 505.

8. White, J. R.; Thomas, E. L. Rubber Chem Technol 1984, 57, 457.

9. The Science and Technology of Rubber, 3rd Ed.; Mark, J. E.; Erman, B; Eirich, F. R., Eds; Elsevier Academic Press: Burlington, MA, 2005.

10. Papadopoulos, I. C.; Thomas, A. G.; Busfield, J. J. C. J Appl Polym Sci 2008, 109, 1900. 